\title{
Interference and Diffraction - A New Theory
}

\author{
Bayyarapu Prasada Rao
}

\begin{abstract}
Interference and diffraction are attributed to wave nature of light .To verify possible alternative cause, experiments conducted from September 1998 to January 2011 at Bayyarapu Physics Laboratories (BPL) Hyderabad India have revealed that these phenomena are due to superfluidity layer adhering to the surfaces of solids and liquids even at room temperature. The superfluid layer, a state between liquid and gas, is formed by ambient gases and density ripples pervade it at all temperatures. The fluid layer is compressible and is displaced by pressure. Patterns in the experiments of Newton's Rings, Fresnel's biprism, Michelson interferometer, oil slicks and soap films are due to density ripples in the fluid layer. Monochromatic light forms bright and dark fringes while white light forms coloured bands owing to reflection, refraction and dispersion of photons in the fluid layer. Density ripples that taper off in the fluid layer are responsible for bending of photons around the solid surface and diffraction pattern.
\end{abstract}

Index Terms-Density ripples, diffraction, fluid layer, interference, surface pattern.

\section{INTRODUCTION}

Dual nature of light is presently accepted description of nature of light by scientific community [1]-[2]. The wave nature of light was postulated to explain the phenomenon of interference and diffraction [3]-[5]. However, extensive experiments conducted afresh have explained that interference and diffraction are on account of fluid layer existing on solid and liquid surfaces even at room temperature. The density ripples in the fluid layer are modified due to mechanical pressure and due to thermal causes.

\section{Methods And MATERIALS}

Interference is mainly studied in the Newton's Rings experiment, Fresnel's biprism experiment, Michelson interferometer, oil slicks and soap films etc.

\section{A. Newton's Rings Experiment}

Newton's Rings [6] comprising alternative bright and dark fringes which taper off from the centre are created at the point of contact when a plano convex lens is placed on the plane glass plate when Sodium light is used to study it. The pattern will have coloured bands when white light (Mercury vapour

Manuscript received August 23, 2012; revised December 3, 2012. This work was supported in part by Higher Education Department, Government of Andhra Pradesh, Osmania University, Hyderabad, Andhra University, Waltair, Jawaharlal Nehru Technological University, Kakinada, Kakatiya University, Warangal, and Dr. Kutikuppala Surya Rao of Visakhapatnam, India.

Bayyarapu Prasada Rao is the with the Departmeny of Physics, Jawaharla Nehru Technological University (JNTU), Kakinada, A.P, India. (e-mail: prasadbayyar@yahoo.co.in). lamp) is used. The diameter of the fringe is supposed to be constant and the fringe diameter and curvature of the lens are used to compute the wave length of Sodium light. When sodium light is used the pattern is attributed to the interference of light coming from the curved portion of the lens and the plane surface below. However, series of experiments have revealed that the Newton's Rings pattern is formed due to density ripples that taper off from centre (point of contact) of lens and glass plate. These ripples are compressions and rarefactions alternatively in the fluid layer with differential density. The compressed portions appear dark due to dispersion and absorption of light and the rarefied portions look bright due to reflection of light when sodium light is used. (Fig. 1 (a) ). However, density gradient of the combined fluid gives rise to coloured bands due to refraction of light when white light is used as shown. (Fig. 1 (b) ).

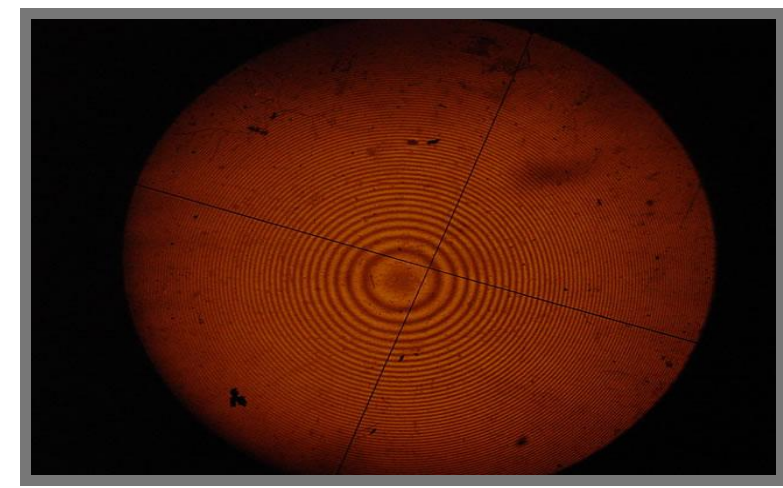

Fig. 1 (a). Newton's rings with sodium lamp

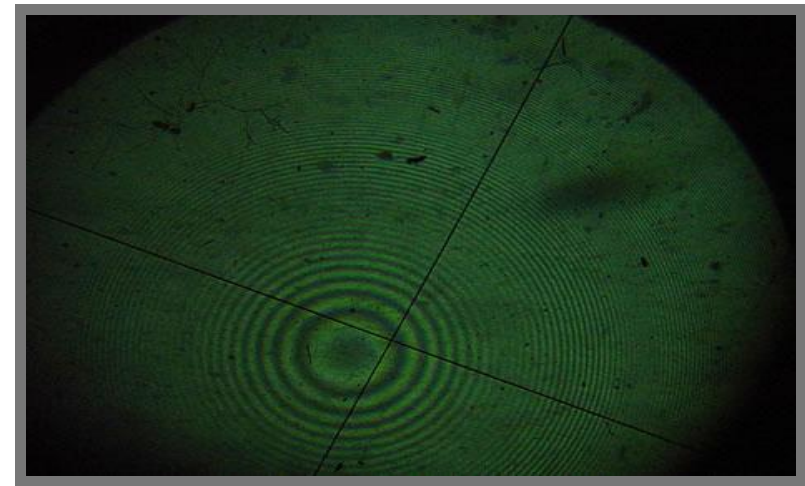

Fig. 1 (b). Newton'S rings with mercury lamp

\section{B. Effect of Pressure}

By exerting pressure on the lens against glass plate, the fluid at the centre is pushed aside giving rise to void at the centre and with enhanced density of fluid surrounding it. Hence, the fringes get modified and become broader as shown in (Fig. 2 (a) ) with Sodium light and (Fig. 2 (b) ) with Mercury vapour lamp. When the pressure is eased the fluid rushes back to cover the portion with the resultant fringes reverting to the original position. 


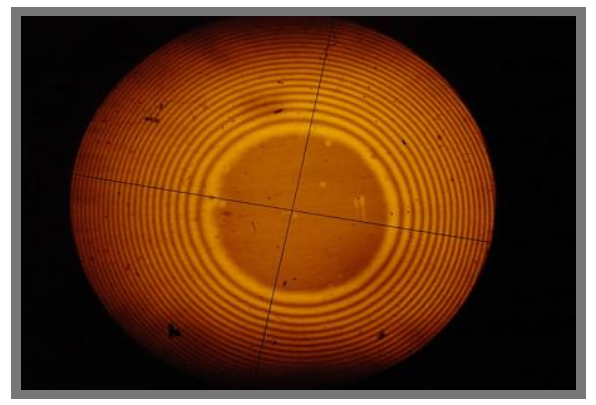

Fig. 2 (a). Fluid displacement at the point of pressure (sodium LAMP)

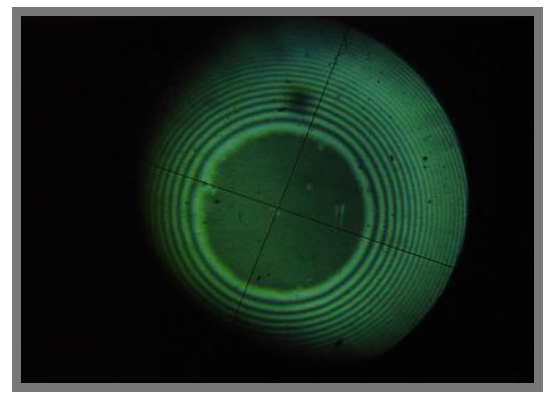

Fig. 2 (b). Fluid displacement at the point of pressure (mercury lamp)

When a piece of human hair is processed between the lens and glass plate, the fluid is divided into two portions giving rise to two distinct patterns as shown in (Fig. 3 (a) ) and (Fig. 3. (b) )with Sodium light and Mercury light respectively.

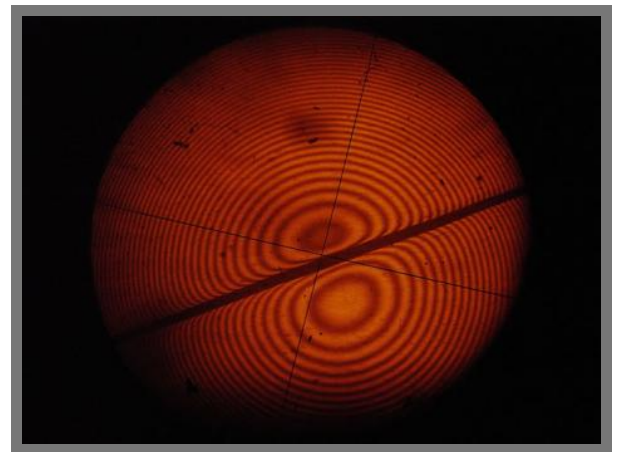

Fig. 3 (a). Division of fluid by a piece of human hair (sodium light)

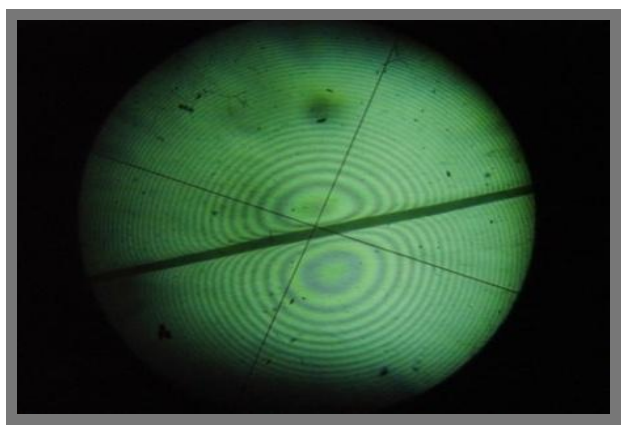

Fig. 3 (b). Division of fluid by a piece of human hair (mercury light)

\section{Effect of Heat}

Any fluid is amenable to heat also. When the Newton's Rings set up is subjected to heat by means of a candle, the fringes appear to be rushing towards the centre and vice versa when heat is withdrawn. When an ice cube is brought in contact underneath the glass plate, the contrary effect is witnessed. Under the influence of heat, density of fluid at the centre gets reduced whereby the fringes get repositioned giving an impression of fringes rushing towards the centre.
When the heat is withdrawn, the density of the fluid is restored to the original position causing repositioning of the fringes. The same effect is witnessed with colour bands when white light is used.

\section{Presence of Fluid Layer on Liquid Surface}

When doughnut shaped oil film is made on glass plate and plano convex lens is placed just above the oil film, it is found Newton's Rings pattern is formed in the glass portion of the centre of the doughnut whereas distorted Newton's Rings pattern is found on the oil film. Pattern with Sodium vapour lamp and Mercury vapour lamp are shown in (Fig. 4 (a) and Fig. 4 (b) ). Different liquids like water diesel etc., give similar results. From these experiments, it is evident that fluid film is present on the liquid surface also.

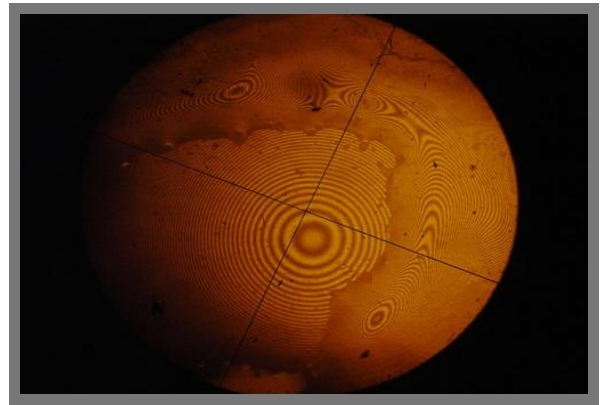

Fig. 4 (a). Newton's rings on glass portion and distorted newton's rings pattern on oil film (sodium light)

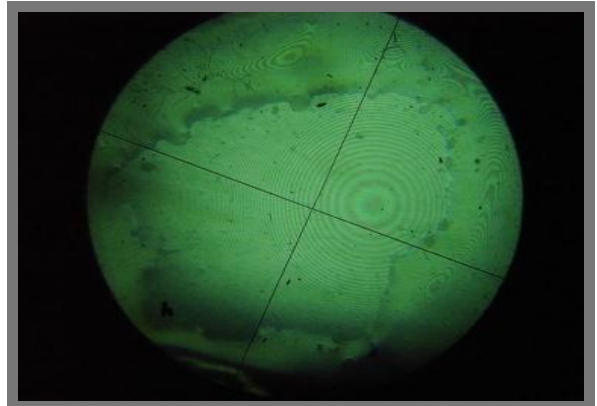

Fig. 4 (b). Newton's rings on glass portion and distorted newton's rings pattern on oil film (mercury light)

\section{E. Surface Pattern}

It is found that part of the fluid layer discussed above, which is very close to the surface of the glass plates, Oil films get compressed due to Vander-wall's forces giving rise to a distinct pattern called surface pattern depending on the thickness and nature of the surface. Above this surface pattern also fluid exists giving rise to different pattern when another surface is brought together. Surface pattern of a thin glass plate is shown in (Fig. 5 (a) ) and polythene sheet is shown in (Fig. 5 (b) ). Pattern above the surface pattern when a lens is placed on a thin glass plate is shown in (Fig .5 (c)).

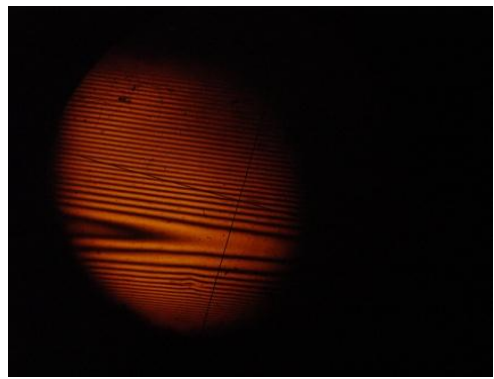

Fig. 5 (a). Surface pattern on a thin glass plate with sodium light 


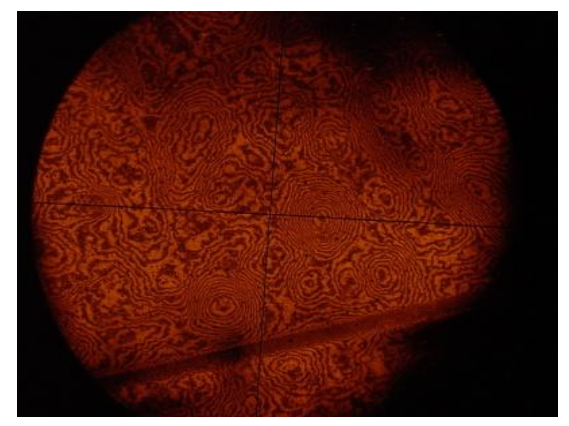

Fig. 5 (b). Surface pattern on a polythene sheet with sodium light

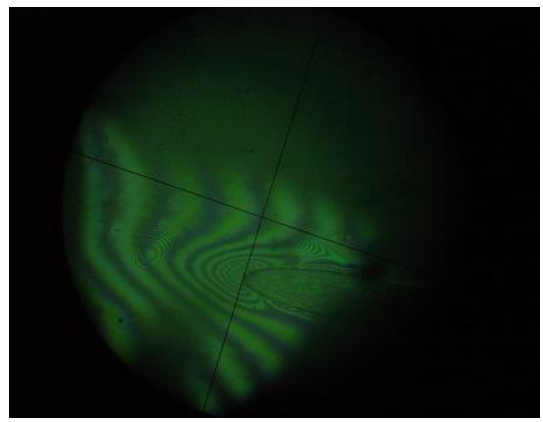

Fig. 5 (c). Pattern above the surface pattern with mercury light

\section{F. Soap Film}

The colours and patterns on the soap film (soap bubbles) [7] and oil slicks are attributed to interference of light from upper surface and lower surface of the film. However, the experiments have revealed that the surface pattern of the fluid layer is responsible for the colours and the pattern in both the cases. In fact, soap film is found to be composite film comprising of the water film sandwiched between the two oil films. As water evaporates, the vapor comes out of the film by making perforations in the oil films. The two perforated oil films of the soap films are shown in (Fig. 6). (The process of perforation formation is captured in the video will be displayed during presentation).

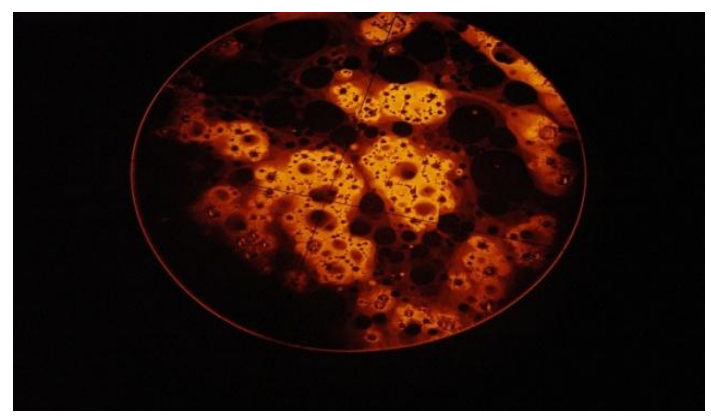

Fig. 6. Two perforated oil films of the soap film

\section{G. Fresnel's Biprism Experiment}

The linear fringes in this experiment are attributed to interference of wavelets emanating from the slit. However, the fringes are found to be the density ripples resent in the fluid layer on the bulge portion that joins the bases of both the prisms of the biprism. When two biprisms are placed at a slant one after another, two distinct portions are witnessed as shown in (Fig. 7). By application of heat, the pattern undergoes modification. (Captured in the video will be displayed during presentation).

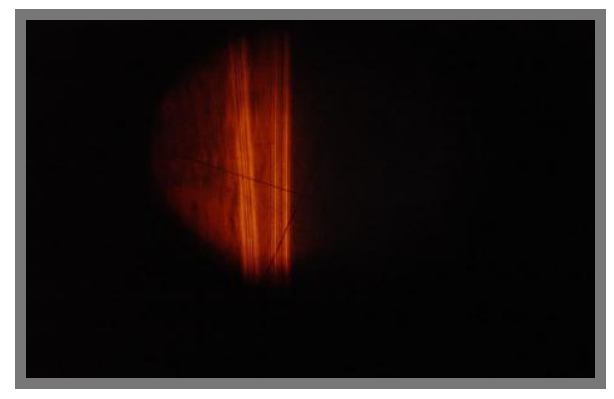

Fig. 7. Two fringe patterns at a slant with Sodium Light.

\section{H. Michelson Interferometer}

The circular fringes in this experiment are attributed to the wavelets emanating from the two silvered mirrors of the Michelson Interferometer. However, actually the surface of the silver mirror has fluid layer which gives rise to surface pattern of circular fringes. Combination of these two fringe patterns is what is actually witnessed.

\section{DIFFRACTION}

Presence of light in the geometrical shadow of the sharp edge of an object when light passes over the edge is called diffraction [8]-[9]The fringe pattern which tapers off from the edge of the surface of an object is also considered as diffraction pattern.[10]-[11] However, from the foregoing experiments, it is evident that the diffraction pattern on the solid surface is due to ripples formed in the fluid layer. The ripples are caused by the thermal vibrations of the surface of the object owing to ambient temperature. Fringe pattern on the surface of an acrylic sheet held in front of the vertical slit illuminated by the Sodium vapour lamp is studied. The acrylic sheet is aligned in such a way that the light reflected from the surface of the sheet gets refracted in the fluid layer and focuses to illuminate the fringes close to the surface. When the acrylic sheet is subjected to heat with a candle, it is found under the influence of heat, the fringe pattern gets disturbed and the light focus shifts away from the surface. On withdrawal of heat the fringe pattern gets reformed and the light focus shifts gradually to the fringes close to the surface. It is found that when heat is transmitted the fluid layer close to the surface gets rarified shifting the light beam away from the surface and the fringes getting modified continuously in the process. On withdrawal of heat, the density of the fluid layer is restored to the original state gradually and fringes are reformed continuously at the same time bringing the light beam back to the original position close to the surface. This is a typical case of density variation of the fluid layer leading to variation in its refractivity and also the fringes varying their position in consonance with the density change. The compressed portions in the ripples of the fluid layer as also the material in the acrylic sheet refracts light giving rise to light in the geometrical shadow of the edge.

\section{DISCUSSION}

The fluid layer on the surface of solids and liquids has the following characteristics. 
1) The fluid layer adheres to the entire surface of the solids and liquids. Its thickness is roughly half a millimeter. The fluid layer is formed by the ambient gases and its density is midway between a gas and a liquid under normal atmospheric conditions.

2) It is compressible, but offers resistance to compression. Under the influence of heat, fluid layer expands. Temperature and pressure thus modify the fringes in the fluid layer. When two surfaces are brought together as the solid- vs - solid, solid- vs - liquid, liquid - vs - liquid, the fluid layers of respective surfaces mingle giving rise to a definite shape of the composite film with distinct fringe pattern.

3) If the glass plate is kept in a high vacuum container and temperature is reduced, the fluid film of each gas gets withdrawn from the surface of the glass plate at the liquefaction temperature of the respective gas. Thus, Oxygen, Nitrogen and Hydrogen fluid films get withdrawn gradually leaving the fluid films of the isotopes of Helium 3 and Helium 4. At $2.17 \mathrm{~K}$, Helium 4 film will be withdrawn because at this temperature Helium 4 gets liquefied. At $0.02 \mathrm{~K}$, Helium 3 film will be withdrawn from the surface as Helium 3 gets liquefied at that temperature. Thus with the disappearance of fluid films from the surface of the glass plate at near absolute zero as discussed above, the ripples that give rise to the diffraction pattern will also disappear. Consequently, neither any Newton's Rings nor interference fringe pattern will be formed under these conditions.

4) In the discharge tube experiment, studying the Cathode Rays, at $10 \mathrm{KV}$ potential and $0.1 \mathrm{~mm}$ pressure [12] the cathode glow was seen moving from the cathode giving rise to the Crook's dark space between the cathode and cathode glow. This can be explained as the fluid layer attached to the cathode captures the electrons and gives the glow. As more electrons are captured in the fluid layer, due to repulsion between the cathode and the negative charge built up in the fluid layer, the fluid layer gets detached and moves forward forming the Crook's dark space. As more electrons are captured the fluid layer gets dissipated gradually due to repulsions of the electrons within the fluid layer.

5) Thus it is seen the superfluity layer formed by Helium at cryogenic temperature continues to adhere to the surface of solids even at room temperature. Superfluity film of Helium, Hydrogen, Nitrogen etc., constitute the fluid layer mentioned above and it remains adhering to the surfaces of solids and liquids even at the room temperature. Conversely any new surface that is formed will have ambient gases adhering to the surface and giving rise to fluid layer.

\section{SUMMARY}

Interference and diffraction patterns are traditionally attributed to the wave nature of light [13]-[14]. However, an alternative theory for interference and diffraction is proposed on the basis of fluid layer adhering to solid and liquid surfaces even at room temperature. This fluid layer caused by the ambient gases is known as 'superfluidity' layer at liquid Helium temperature. Fluid layer is a state between gas and liquid and density ripples in the fluid layer is known as diffraction pattern. The pattern arising due to density ripples in the combination of fluid layers on account of two surfaces brought together is known as interference. Refraction, absorption and scattering of photons of light impinging on the density ripples are perceived as interference and diffraction patterns. Thus interference and diffraction are explained by a new theory without invoking the Wave Theory of light.

\section{ACKNOWLEDGMENT}

The Author thanks the Higher Education Department, Government of Andhra Pradesh, Osmania University, Hyderabad, Andhra University, Waltair, Jawaharlal Nehru Technological University, Kakinada, Kakatiya University, Warangal, and Dr. Kutikuppala Surya Rao of Visakhapatnam, India.

\section{REFERENCES}

[1] L. D. Brogue, Matter and light, 1939

[2] The Wrolds of David Darling. The Internet Encyclopedia of Science. [Online] Available: http://www.daviddarling.info/encyclopedia/W/wave-particle_duality. html.

[3] D. M. Greenberger and A. Yasin. (1988). Simultaneous wavge and particle knowledge in a neutron interferometer. Physics Letters A 128 , 391-4, [Online]. Available: http://www.arstechnica.com/science/2012/05/disentangling-the-waveparticle-duality-in-the-double-slit-experiment/

[4] P. Mittelstaedt, A. Prieur, and R. Schieder, "Unsharp particle-wave duality in a photon split-beam experiment," Foundations of Physics, vol. 17 , no. 9, pp. 891-903, 1987.

[5] B. D. Pintado and C. Maria. Updating the wave-particle duality. Presented at 15th UK and European Meeting on the Foundations of Physics, Leeds, UK. [Online]. Available: http://www.philsci-archive.pitt.edu/archive/00003568/.

[6] F. A. Jenkins and H. E. White, Fundamentals of Optics, 4th edition McGraw Hill Book Co., 1981, pp. 228.

[7] F. W. SeARS, Optics, Addison-Wesley Publ. Co., 1949, pp. 203-209, F. W. Sears and M. W. Zemansky, UniversityPhysics,Addison, Wesley Publ.Co., 1976, pp. 600

[8] B. E.Wolf, Principles of optics, 6 edition, Pergamon Press, 1989.

[9] D. Morin, Interference and diffraction, version 1, June 25, 2010.

[10] O. Nairz, B. Brezger, M. Arndt, and A. Zeilinger. (2001). Diffraction of Complex Molecules by StructuresMadeofLight. Phys. Rev. Lett.. [Online]. http://www.prl.aps.org/abstract/PRL/v87/i16/e160401

[11] T. A. Littlefield and N. Thorley, Atomic and Nuclear Physics, Van Nostrand Reinhold, London, 1971, pp. 21-22.

[12] C. Brukner and A. Zeilinger, "Young's experiment and the finiteness of information," Philos. Trans. R.Soc. Lond, 2002, vol. 360, pp. 1061-1069.

[13] New Scientist: Quantum wonders: Corpuscles and buckyballs. (2010). [Online]. Available: http://www.newscientist.com/article/mg20627596.100-quantum-wond ers-corpuscles-and-buckyballs.html

[14] M. Arndt, O. Nairz, J. V. Andreae, C. Keller, G. V. D. Zouw, and A. Z Nature, Wave-particle duality of C60 molecules, 14 October 1999.

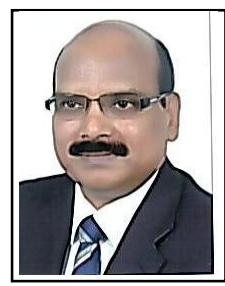

Bayyarapu Prasada Rao was born in the City of Vijayawada of Andhra Pradesh in India on 11th September, 1955. He passed out from Andhra Loyola College, Vijayawada of Andhra Pradesh with distinction in B.Sc., (Physics Main) in 1975 and M.Sc., (Masters in Science - Physics) from the Indian Institute of Technology, Madras, Chennai, India in 1977. He passed Indian Civil Services (IPS) with Physics as Main subject in 1979. 
He established Bayyarapu Physics Laboratories (BPL), Hyderabad, India and conducted experiments on Wave theory on nature of light from 1988 to January, 2011. As a Visiting Professor, Physics Department, Jawaharlal Nehru Technological University, Kakinada, India, with the Kakatiya University, Warangal, India, has been extensively conducting workshops, delivering lectures to Post-graduate students of Physics and guiding research scholars. He presented papers in prestigious conferences such as Indian Science Congress, Bangalore, which was addressed by the then Hon'ble President of India, Dr.A.P.J.Abdul Kalam, National conference on Spectro Physics in Chennai, India etc., His works were published in several National and International Journals. He widely toured and addressed International Association of Public Transport at France, Hongkong in 2010 and Dubai in 2011.His publications viz; 1. Wave theory of Light - A historical blunder?? Bayyarapu Prasada Rao; Proceedings of Indian Science Congress - Vol.Jan
2003. 2. New Light on LIGHT - Bayyarapu Prasada Rao Atti Della “FONDAZIONE GIORGIO RONCHI' Anno LIX 2004, Italy.3. Discovery Disputes Wave Theory of Light - Bayyarapu Prasada Rao; India Tribune, Chicago, U.S.A., March 30, 2012; have been acclaimed globally as path breaking research in the area of Nature of Light. His current research is on Big Bang theory; Dark matter, Dark energy, Evolution of Universe, Neutrino Physics. Professor Bayyarapu Prasada Rao is a Member in Indian Science Congress, Director General of Police, Anti-Corruption Bureau, Government of Andhra Pradesh; Board of Governors, Holy Mary Institutions of Education, Board of Studies, Department of Physics, Kakatiya University. He won Indian Police Medal for meritorious service in 1997 and President's Police Medal for distinguished service in 2006 from His Excellency, President of India. 